\title{
Fibroblast-derived exosomal microRNA-369 potentiates migration and invasion of lung squamous cell carcinoma cells via NF1-mediated MAPK signaling pathway
}

\author{
LIPING GUO $^{1 *}$, BAOLI LI ${ }^{2 *}$, JIANJUN YANG ${ }^{3}$, JUAN SHEN ${ }^{4}$, JINSHAN JI ${ }^{5}$ and MEIJING MIAO ${ }^{6}$ \\ Departments of ${ }^{1}$ Pathology and ${ }^{2}$ Pharmacology, Medical College of Yan'an University; \\ ${ }^{3}$ Department of Interventional Radiology, Affiliated Hospital of Yan'an University; Departments of ${ }^{4}$ Imaging, \\ ${ }^{5}$ Preventive Medicine and ${ }^{6}$ Nursing, Medical College of Yan'an University, Yan'an, Shaanxi 716000, P.R. China
}

Received January 10, 2020; Accepted April 16, 2020

DOI: $10.3892 / \mathrm{ijmm} .2020 .4614$

\begin{abstract}
Cancer-associated fibroblasts (CAFs) exhibit tumor-stimulating properties and are associated with poor survival in several types of cancer, making them potential therapeutic targets. The present study aimed to determine whether CAFs were associated with cell migration and invasion in lung squamous cell carcinoma (LUSC), as well as their association with microRNA-369 (miR-369) in these processes. Firstly, the changes of the malignant biological behavior were observed by treating the LUSC cells with the CAFs-derived extracellular vesicles (CAFs-EVs). Subsequently, the differentially expressed miRNAs in the cells treated with CAFs-EVs were analyzed by microarray analysis. Following inhibition of miR-369 expression in CAFs-EVs, LUSC cells were co-cultured, and the malignant biological behavior of the cells was re-examined. Then, through bioinformatics analysis and verification, the mRNA targets of miR-369 and the corresponding downstream signaling pathway were screened out. Finally, the effects of CAFs-EVs on the growth and metastasis of LUSC were demonstrated by in vivo tumor formation and metastasis experiments. It was identified that miR-369 was expressed at a relatively high level in the CAFs-EVs. Neurofibromin-1 (NF1) was hypothesized as a direct target of miR-369 in LUSC. Also, the overexpression of miR-369 activated the mitogen-activated protein kinase signaling pathway by interacting with NF1, consequently potentiating LUSC cell growth. The present study provided novel insights
\end{abstract}

Correspondence to: Ms. Meijing Miao, Department of Nursing, Medical College of Yan'an University, 38 Guanghua Road, Baota, Yan'an, Shaanxi 716000, P.R. China

E-mail: miaomeijing11071@163.com

${ }^{*}$ Contributed equally

Key words: cancer-associated fibroblasts, lung squamous cell carcinoma, microRNA-369, neurofibromin-1, mitogen-activated protein kinase signaling pathway, migration, invasion into the action of miR-369 in CAFs-EVs in controlling LUSC cell migration, invasion and tumorigenesis, and identified miR-369 in CAFs-EVs as an important prognostic marker and therapeutic target.

\section{Introduction}

As one of the most prevalent types of human cancer, lung cancer is responsible for one-quarter of cancer-associated mortalities worldwide (1). Lung cancer rates and trends differ considerably according to sex, age, race, socioeconomic status and geography, due to alterations in historical smoking patterns (2). Non-small cell lung cancer is divided into three different subtypes: Lung adenocarcinoma; lung squamous cell carcinoma (LUSC); and large cell lung carcinoma. LUSC contributes to $\sim 30 \%$ of the total lung cancer cases and results in $\sim 400,000$ mortalities each year worldwide (3). There have been many advances in the development of molecular lung adenocarcinoma-targeted therapies, while limited progress has been made in the development of treatments for LUSC, with the exception of immunotherapy (4).

Cancer-associated fibroblasts (CAFs) have been documented to stimulate cancer cell proliferation and invasion, thereby supporting tumorigenesis (5). CAFs are defined by the acquisition of an $\alpha$-smooth muscle actin ( $\alpha$-SMA)-positive, 'activated' myofibroblast phenotype (6). CAFs and cancer cells have been revealed to have the ability to release exosomal microRNAs (miRNAs) to exert effects over each other, and the extracellular vesicles (EVs) can be categorized into three types, on the basis of biogenesis and size: Exosomes (30-100 nm); microvesicles (100-1000 nm); and oncosomes $(1-10 \mu \mathrm{m})(7)$. Exosomes could modulate local and systemic cell communication through the transmission of information via microRNAs, proteins, mRNAs and other substances (8). For example, exosomal miR-382-5p released by CAFs promoted the cell migration and invasion in oral squamous cell carcinoma (9). Notably, overexpression of miR-369-3p was identified in cisplatin-resistant non-small cell lung cancer (NSCLC) tissues and cells and associated with the malignancy of these cells (10). Using StarBase (11) and TargetScan (12), miR-369 was predicted to bind to neurofibromin 1 (NF1) in 
LUSC cells in the current study. NF1, located on chromosome 17q11.2, is an important tumor suppressor and encodes a rat sarcoma (Ras)-GTPase activating protein identified as neurofibromin (13). Glomus cells with inactivated NF1 exhibited elevated Ras-mitogen-activated protein kinase (MAPK) signals (14). Neurofibromin accelerates the transfer of the Ras family to their inactive state and acts as a suppressor of the MAPK signaling pathway (15). In addition, NF1 deficiency facilities murine Kirsten rat sarcoma viral oncogene homolog gene-driven tumorigenesis of lung adenocarcinomas (16), which may validate the functional activity of NF1 mutations in NSCLC and the efficacy of targeted suppression of downstream Ras signaling pathway (17). In the present study, miR-369 secreted by CAF-EVs is reported to activate the MAPK signaling pathway by downregulating NF1, initiating development and progression of LUSC.

\section{Materials and methods}

Tissue collection. A total of 52 paired LUSC and matching paratumor tissues ( $>5 \mathrm{~cm}$ from the LUSC tissues) were harvested by resection from patients diagnosed and treated in the Affiliated Hospital of Yan'an University from November 2012 to August 2013. The cohort consisted of 39 males and 13 females, ranging from 49-79 years, with an average age of $64.9 \pm 7.3$ years. Follow-up was conducted every 3 months for 5 years. All the patients were diagnosed with LUSC on the basis of histopathological evaluation and had complete clinical data. No patients had been subjected to radiotherapy or chemotherapy prior to surgical treatment. Patients combined with chronic system disorders, such as acquired immune deficiency syndrome, autoimmune diseases and diabetes, or other malignancies were excluded. All human tissue donors provided written informed consent prior to tissue donation. The study protocol was approved by the Institutional Review Board of Affiliated Hospital of Yan'an University.

Bioinformatics analysis. To screen differentially expressed miRNAs in cells co-cultured with EVs, miRCURY LNA ${ }^{\mathrm{TM}}$ Universal RT microRNA PCR Human panel (Exiqon; Qiagen $\mathrm{GmbH}$ ) was used. miRNAs differentially expressed in H520 cells co-cultured with PBS and EVs were analyzed, according to the manufacturer's protocol. One-way analysis of variance was applied for comparisons with threshold settings as: $\mathrm{P}<0.05$ and fold-change $>2.5$ or $<-2.5$. StarBase (http://starbase.sysu.edu.cn/) platform was subsequently used to detect miR-369 expression in lung cancer patients in The Cancer Genome Atlas (TCGA) database (https://cancergenome.nih.gov/) (18). Subsequently, StarBase and TargetScan (version 7.2; http://www.targetscan.org/vert_72/) were used to predict the targeting mRNAs of miR-369. The targeting mRNAs of miR-369 were subjected to Kyoto Encyclopedia of Genes and Genomes enrichment analysis (https://www.kegg. $\mathrm{jp} /$ ) (19) using a bioinformatics software DAVID (version 6.7; https://david.ncifcrf.gov/).

Culture and identification of CAFs. The CAFs were isolated and extracted from surgically removed tissues from 6 patients with LUSC treated in the Affiliated Hospital of Yan'an University from July to December 2017. Subsequent to detachment through collagenase/trypsin, the cells were cultured until they reached $>85 \%$ confluence, and then detached with trypsin again for passage. Isolated CAFs were maintained in Dulbecco's modified Eagle's medium (DMEM)-F12 (Sigma-Aldrich; Merck KGaA) containing 10\% FBS depleted of exosomes (exo-FBS; System Biosciences, LLC), blocked with $1 \%$ bovine serum albumin (Beijing Solarbio Life Sciences Co., Ltd.) for $30 \mathrm{~min}$ at $37^{\circ} \mathrm{C}$ and treated with $1 \mathrm{X}$ antibiotic-antimycotics (Thermo Fisher Scientific, Inc.). The CAFs ( $1 \times 10^{6}$ cells $\left./ \mathrm{ml}\right)$ were stained for 30 min with phycoerythrin-conjugated monoclonal antibodies against prolyl endopeptidase FAP (FAP; cat. no. ab207178; 1:50; Abcam), vimentin (cat. no. ab92547; 1:50; Abcam) and $\alpha$-SMA (cat. no. ab32575; $1: 50$; Abcam) at $37^{\circ} \mathrm{C}$ in the dark. Following phosphate buffered saline (PBS) washes, the cells were examined using a FACSCaliburTM flow cytometer (BD Biosciences) and analyzed using FlowJo V11.0 (BD Biosciences).

Extraction and identification of EVs. Following CAF identification, the cells between passages 3 and 12 were used for EV extraction. EVs were extracted from CAFs culture medium and centrifuged for $10 \mathrm{~min}$ at $3,000 \mathrm{xg}$ at $4^{\circ} \mathrm{C}$. The medium was then subjected to two rounds of centrifugation to remove cell debris, firstly at $3,000 \times \mathrm{g}$ for $10 \mathrm{~min}$ and then at $10,000 \mathrm{x} \mathrm{g}$ for $30 \mathrm{~min}$ at $4^{\circ} \mathrm{C}$. Subsequently, $1 \mathrm{ml}$ culture medium was centrifuged at $4^{\circ} \mathrm{C}$ at $100,000 \times$ g for $2 \mathrm{~h}$ using Class H, R, and S Preparative Ultracentrifuges with a Type 50.4 Ti Rotor (Beckman Coulter, Inc.) to obtain the precipitate. This precipitate was washed with PBS, then filtered through a $0.22 \mu \mathrm{m}$ filter, and then ultra-centrifuged at $4^{\circ} \mathrm{C}$ for $2 \mathrm{~h}$ at $100,000 \mathrm{xg}$ to precipitate the EVs. The precipitate resuspended in $1 \mathrm{ml}$ PBS or lysis buffer (Beyotime Institute of Biotechnology) was used for protein measurement. The extracted EVs were then maintained at $-80^{\circ} \mathrm{C}$ or treated with $10 \%$ sodium dodecyl sulfate (SDS) to damage EV membrane structure.

The isolated EV suspension was resuspended and dropped onto the sealing membrane. The resuspended pellets were deposited on electron microscopy grids. The grids were adsorbed for $20 \mathrm{~min}$, transferred to glutaraldehyde droplet for about $5 \mathrm{~min}$, and then transferred to the appropriate washing buffer. Repeat transfers to fresh drops of washing buffer was performed seven times. Then, the grids were dried and transferred to a $4 \%$ hydrogen peroxide droplet for $10 \mathrm{~min}$, and then transferred to a $1 \%$ methyl cellulose droplet for $5 \mathrm{~min}$ (all steps were carried out on ice). After being air-dried for 30 min, images were captured using a transmission electron microscope $(80 \mathrm{kV})$ and analyzed by DigitalMicrograph 3.9 (magnification, x40,000; Gatan, Inc.).

CAFs were transfected with $10 \mathrm{nM}$ miR-369 inhibitor (5'-AGAUCGACCGUGUUAUAUUCGC-3') or scramble inhibitor (5'-GGUUGAUCUUUUCUCAGUA-3'; Tebu-bio) using Oligofectamine (Invitrogen; Thermo Fisher Scientific, Inc.). A total of $6 \mathrm{~h}$ after transfection, the cells were cultured in fresh DMEM-F12 supplemented with 1\% A/A and 10\% exo-FBS for 1 day. The media were harvested for exosome preparation. The H520 and SK-MES-1 cells were then co-cultured with exosomes at a concentration of $1 \times 10^{9}$ particles $/ \mathrm{ml}$. The inhibitors or scramble inhibitors utilized were synthesized by Shanghai GenePharma Co., Ltd. 
Western blot analysis. Total protein was extracted from CAFs-derived exosomes or supernatant using radioimmunoprecipitation assay lysis buffer (Beyotime Institute of Biotechnology). Briefly, $40 \mathrm{mg}$ protein were loaded on each lane, separated using 10\% SDS-PAGE and transferred onto polyvinylidene fluoride membranes. Following blocking with $5 \%$ skim milk at room temperature for $30 \mathrm{~min}$, the membranes were subsequently incubated with the primary antibodies (all 1:1,000) against CD9 antigen (cat. no. ab92726; Abcam), CD63 antigen (cat. no. 55051; Cell Signaling Technologies, Inc.), tumor susceptibility gene 101 protein (cat. no. ab125011; Abcam), heat shock protein 70 (HSP70; cat. no. 4873; Cell Signaling Technologies, Inc.), CD81 antigen (cat. no. 10037; Cell Signaling Technologies, Inc.) overnight at $4^{\circ} \mathrm{C}$, and with horseradish peroxidase (HRP)-labeled goat anti-rabbit IgG (cat. no. G-21234; Invitrogen; Thermo Fisher Scientific, Inc.; 1:5,000) at room temperature for $2 \mathrm{~h}$. Blots were visualized using chemiluminescence detection (GE Healthcare) and analyzed by ImageJ 1.8.0 software (National Institutes of Health). Glyceraldehyde-3-phosphate dehydrogenase (GAPDH; cat. no. D190090; Shanghai Sangon Biological Engineering Technology \& Services Co., Ltd.) was applied as an internal reference.

Cell culture and grouping. Human LUSC H520 and SK-MES-1 cell lines were obtained from the Institute of Biochemistry and Cell Biology, Shanghai Institutes for Biological Sciences, Chinese Academy of Sciences. The aforementioned cells were seeded into cell culture dishes at $1 \times 10^{5}$ cells $/ \mathrm{cm}^{2}$ and maintained in Roswell Park Memorial Institute (RPMI)-1640 (Gibco; Thermo Fisher Scientific, Inc.) supplemented with 10\% FBS (Gibco; Thermo Fisher Scientific, Inc.) for $48 \mathrm{~h}$ at $37^{\circ} \mathrm{C}$ with $5 \% \mathrm{CO}_{2}$. When the cells reached $80-90 \%$ confluence, they were detached with $0.25 \%$ trypsin (Gibco; Thermo Fisher Scientific, Inc.), and then passaged.

Subsequently, H520 cells and SK-MES-1 cells at a confluence $>75 \%$ were co-cultured with $20 \mu \mathrm{l}$ PBS and $20 \mu \mathrm{M}$ exosomes from un-transfected cells or cells transfected with miR-369 inhibitor or inhibitor control.

Reverse transcription-quantitative PCR (RT-qPCR). The total RNA was extracted from tissues and cells using RNAiso Plus (Takara Bio, Inc.) or TRIzol ${ }^{\circledR}$ LS Reagent (Takara Bio, Inc.). Subsequently, $10 \mathrm{mg}$ RNA was subjected to a 30-min at $10 \mathrm{~V} / \mathrm{cm}$ voltage using $2 \%$ agarose gel at room temperature to verify the reliability of RNA. Then, a PrimeScript TM RT kit (Takara Bio, Inc.) was used to perform the RT strictly according to the protocol of the manufacturer. qPCR analyses were performed by 30 cycles of denaturation at $95^{\circ} \mathrm{C}$ and annealing at $45^{\circ} \mathrm{C}$, followed by extension at $72^{\circ} \mathrm{C}$ using SYBR Premix Ex Taq (Takara Bio, Inc.). The obtained quantification data were normalized to the U6 expression. The sequences of the primers used were as follows: miR-369 forward, 5'-GGGACC CAGTTCAAGTAATTCAGG-3'; miR-369 reverse, 5'-TTT GGCACTAGCACATT-3'; U6 forward, 5'-CTCGCTTCGGCA GCACA-3'; and U6 reverse 5'-AACGCTTCACGAATTTG CGT-3'. Data were determined using the $2^{-\Delta \Delta C q}$ method (20).

Cell counting kit-8 (CCK-8). Cells in a logarithmic growth phase were detached with trypsin, diluted into a single cell suspension at $1 \times 10^{4}$ cells/ml using RPMI-1640 containing $10 \%$ FBS, and then added to a 96-well plate, with $2 \times 10^{3}$ cells each well in $200 \mu \mathrm{l}$ solution. A blank well free of cells containing the culture medium was used as a blank control. A total of 5 identical wells were prepared for each group. Following inoculation, the cells were grown under $5 \% \mathrm{CO}_{2}$ at $37^{\circ} \mathrm{C}$ for 3 days. At $0,24,48$ and $72 \mathrm{~h}$, cells in each well were supplemented with $20 \mu \mathrm{l}$ CCK-8 solution $(5 \mathrm{mg} / \mathrm{ml})$ and cultured for an additional $4 \mathrm{~h}$. Subsequently, $200 \mu \mathrm{l}$ dimethyl sulfoxide was added to the cells in each well and oscillated for $10 \mathrm{~min}$ to thoroughly dissolve the crystals, followed by the measurement of optical density (OD) value at the wavelength of $490 \mathrm{~nm}$.

5-Ethynyl-2'-deoxyuridine (EdU) staining. The DNA replication capacity of H520 and SK-MES-1 cells at passage 3 was detected by a Cell-light EdU luminous detection kit (Guangzhou RiboBio Co., Ltd.) according to the instructions of the manufacturer. A total of 5 visual fields were randomly selected for analysis using a FSX100 fluorescence microscope (magnification, x200; Olympus Corporation). Blue fluorescence represented total cells, while the red fluorescence denoted the replicating cells that had incorporated the EdU. Finally, the percentage of EdU-positive cells was calculated.

Immunofluorescence. Cells were fixed for $15 \mathrm{~min}$ in $4 \%$ paraformaldehyde at $4{ }^{\circ} \mathrm{C}$ and treated for $20 \mathrm{~min}$ with $0.5 \%$ Triton X-100. Antibodies against epithelial-cadherin (1:200; cat. no. ab92552; Abcam) and neural-cadherin (1:100; cat. no. ab133504; Abcam) were utilized as primary antibodies, while Alexa Fluor 594-labeled goat anti-rabbit (1:5,000; cat. no. ab150088; Abcam) used as a secondary antibody. The cells were subsequently counter-stained with 4',6-Diamidino-2-Phenylindole and detected using a Leica DM3000 fluorescence microscope (magnification, x400; Leica Microsystems, Inc.).

Transwell assays for cell migration and invasion. For the invasion assays, $60 \mu \mathrm{l}$ Matrigel (BD Biosciences) diluted in $300 \mu \mathrm{l}$ serum-free medium at $4^{\circ} \mathrm{C}$ was added under aseptic condition and cultured in the apical chamber for $30 \mathrm{~min}$. RPMI-1640 medium $(30 \mu \mathrm{l})$ was then added in the apical chamber and cultured in the $5 \% \mathrm{CO}_{2}$ incubator at $37^{\circ} \mathrm{C}$ for $30 \mathrm{~min}$. Following a 10 -min centrifugation $\left(160 \mathrm{x}\right.$ g at $\left.4^{\circ} \mathrm{C}\right)$, the cell suspension was diluted at a density of $5 \times 10^{5}$ cells $/ \mathrm{ml}$. Next, $500 \mu 1$ RPMI-1640 medium containing 10\% FBS was placed in the Transwell basolateral chamber, with $200 \mu \mathrm{l}$ cell suspension in the apical chamber. The plate was placed in a $37^{\circ} \mathrm{C}$ incubator under $5 \% \mathrm{CO}_{2}$ for $48 \mathrm{~h}$ and then subjected to $1 \%$ crystal violet staining for $10 \mathrm{~min}$ at room temperature. The cells that had not invaded were removed with cotton balls, and images were captured under a light microscope (magnification, $\mathrm{x} 200$ ) to calculate the number of cells. The migration capacity was assessed using a Transwell assay without the addition of Matrigel in the apical chamber. During this protocol, the chambers were removed for staining after $24 \mathrm{~h}$.

ELISAs. NF1 (cat. no. HEN020), MAPK/ERK kinase 1 (MEK1; cat.no. HEM016), pMEK1 (cat.no.HEM017), ERK1/2 (cat. no. HEE013) and pERK1/2 (cat. no. HEE014) ELISA kits 
(Shanghai Bogoo Biological Technology Co., Ltd.) were used in strict accordance with the protocols of the manufacturer. Briefly, the cells were lysed with radioimmunoprecipitation assay lysis buffer containing proteinase inhibitors. Following centrifugation, the supernatants were added into the 96-well plate, followed by blocking for $2 \mathrm{~h}$ with a blocking buffer at room temperature. Antibodies against NF1, MEK1, pMEK1, ERK1/2 and pERK1/2 were added to the medium and incubated for $1 \mathrm{~h}$. Following sequential introduction of substrate solution and termination solution, the OD value at $450 \mathrm{~nm}$ of each well was measured using an automated microplate reader (BioTek Instruments, Inc.) within $30 \mathrm{~min}$.

Dual-luciferase assay. StarBase (http://starbase.sysu.edu. $\mathrm{cn} /$ ), a bioinformatic software (11), was chosen to predict the binding sequence of miR-369 and NF1-3' untranslated region (UTR). NF1 wild type (WT) and 3'UTR binding sequence mutant (MT) were synthesized by Shanghai Sangon Biological Engineering Technology \& Services Co., Ltd. and inserted into pMIR-REPORTTM (Thermo Fisher Scientific, Inc.). WT plasmids, MT plasmids and miR-369 mimic and miRNA negative control (NC) were co-transfected into $293 \mathrm{~T}$ cells using a Lipofectamine ${ }^{\circledR} 3000$ transfection kit (Invitrogen; Thermo Fisher Scientific, Inc.) following the manufacturer's instructions. The cells were lysed $24 \mathrm{~h}$ after transfection, and the luciferase activity was detected by a Dual-Luciferase Reporter Assay System (Promega Corporation), as previously described (21), and normalized to Renilla luciferase activity.

Tumor xenograft model. A total of 40 specific-pathogenfree-grade BALB/c nude mice (age 4-6 weeks, weight $20 \pm 2 \mathrm{~g}$ ) were purchased from Beijing Vital River Laboratory Animal Technology Co., Ltd. [permit number: SCXK (Beijing) 2015-0001], and then randomized into 4 groups, with 10 animals in each group. A total of $4 \times 10^{6} \mathrm{H} 520$ and SK-MES-1 cells in $100 \mu 1$ saline were injected into nude mice subcutaneously. All mice were housed in a temperature- $\left(22 \pm 1^{\circ} \mathrm{C}\right)$ and humidity-controlled $(45-55 \%)$ room with a 12:12 h light: Dark cycle and ad libitum access to chow and drinking water. No mice were died during the treatment process. The tumor volume (V) was measured every week after 7 days of injection and calculated using calipers with the formula: $\mathrm{m}_{1}^{2} \mathrm{xm}_{2} \mathrm{x} 0.5236$, in which $\mathrm{m}_{1}$ represents the shortest diameter and $\mathrm{m}_{2}$ the longest diameter (22). A total of 28 days following implantation, the mice were euthanized, and tumors were excised for histological analysis. For mice receiving EVs treatment, $100 \mu \mathrm{l} \mathrm{EVs}(20 \mu \mathrm{M})$ were injected every 5 days to the site of subcutaneous tumors as previously described (23). At the end of the experiment (28 days after the injection of cells), mice were euthanized by intraperitoneal injection of $1 \%$ pentobarbital sodium at $100 \mathrm{mg} / \mathrm{kg}$. Following euthanasia, animal death was confirmed by observing the lack of heartbeat, respiratory arrest, pupil dilation, and lack of nerve reflex.

Immunohistochemical staining. The tumor tissues from each group were paraffin-embedded, dewaxed (in xylene I and xylene II for $20 \mathrm{~min}$, respectively) and hydrated (twice in anhydrous ethanol, once in 95\% ethanol and once in $80 \%$ ethanol; 5 min each time). A total of 5 sections were collected from each tumor. The sections were then incubated with $\mathrm{H}_{2} \mathrm{O}_{2}$ for
$15 \mathrm{~min}$ at room temperature to eliminate the activity of endogenous peroxide. Following sealing with normal goat serum at room temperature for $15 \mathrm{~min}$, the sections were probed at $4^{\circ} \mathrm{C}$ overnight with $50 \mu 1$ rabbit anti-human antibody against proliferation marker protein Ki-67 (Ki-67; 1:500; cat. no. ab15580; Abcam) and the HRP-conjugated secondary goat anti-rabbit antibody against IgG (1:500; cat. no. GTX213110-01; GeneTex, Inc.) at $37^{\circ} \mathrm{C}$ for $15 \mathrm{~min}$. Following treatment with $40 \mu \mathrm{l}$ HRP-conjugated streptomyces ovalbumin working solution at $37^{\circ} \mathrm{C}$ for $15 \mathrm{~min}$, the sections were stained with $0.01 \mathrm{M}$ $3,3^{\prime}$-diaminobenzidine at room temperature for $30 \mathrm{~min}$. Finally, the sections were counterstained with $2 \%$ hematoxylin in ethanol at room temperature for $30 \mathrm{sec}$, dehydrated and mounted. The cells with brownish yellow or brown nucleus were regarded as Ki-67-positive. A total of 5 fields were chosen randomly and observed under a light microscope (magnification, $\mathrm{x} 200$ ), and the positive rate was calculated as the number of positive cells/the number of total cells x $100 \%$.

In vivo metastasis test. A total of $4 \times 10^{6} \mathrm{HT} 29$ and SW116 cells were injected into 40 mice through the tail veins ( $\mathrm{n}=10 /$ group). A total of $100 \mu \mathrm{l}$ EVs $(20 \mu \mathrm{M})$ were injected every 5 days with a total of 8 injections through the tail veins as previously described (24). No mice were died during the process. In addition, the health and behavior of mice were observed every day in the experiment, and the mice exhibited good appetite, normal activity and good mental condition prior to the end of the experiment. At the end of the experiment, 45 days after the injection of cells, mice were euthanized by intraperitoneal injection of $1 \%$ pentobarbital sodium at $100 \mathrm{mg} / \mathrm{kg}$. Following euthanasia, animal death was confirmed by observing the absence of a heartbeat, respiratory arrest, pupil dilation, and lack of nerve reflex. The lung and liver tissues were removed for hematoxylin \& eosin staining as previously described (25).

Statistical analysis. Statistical analyses were performed with SPSS 21.0 (SPSS, Inc.). Kolmogorov-Smirnov testing was used to assess normality. The results were expressed as mean \pm standard deviation (SD). Statistical analysis of differences between experimental groups was performed using paired two-tailed t-tests, whereas differences among multiple groups were analyzed by one-way or two-way analysis of variance with Tukey's post hoc test. Fisher's exact test was used to compare the enumeration data. $\mathrm{P}<0.05$ was considered to indicate a statistically significant difference.

\section{Results}

Identification of the CAF-EVs. Flow cytometry was first used to identify the presence of CAFs surface markers $\alpha$-SMA and FAP, and the fibroblast marker vimentin, in the cells (Fig. 1A). Then, the CAF-derived EVs were identified by transmission electron microscopy, nanoparticle tracking analysis and western blot analysis assays. The results showed that the extracted EVs corresponded to the definition of the EVs of the CAFs from the Minimal information for studies of extracellular vesicles 2018 (MISEV2018) guidelines (26) (Fig. 1B-D).

Malignancies of LUSC cells are promoted by CAF-derived EVs treatment. Then, a CCK-8 kit was used to evaluate the cells 

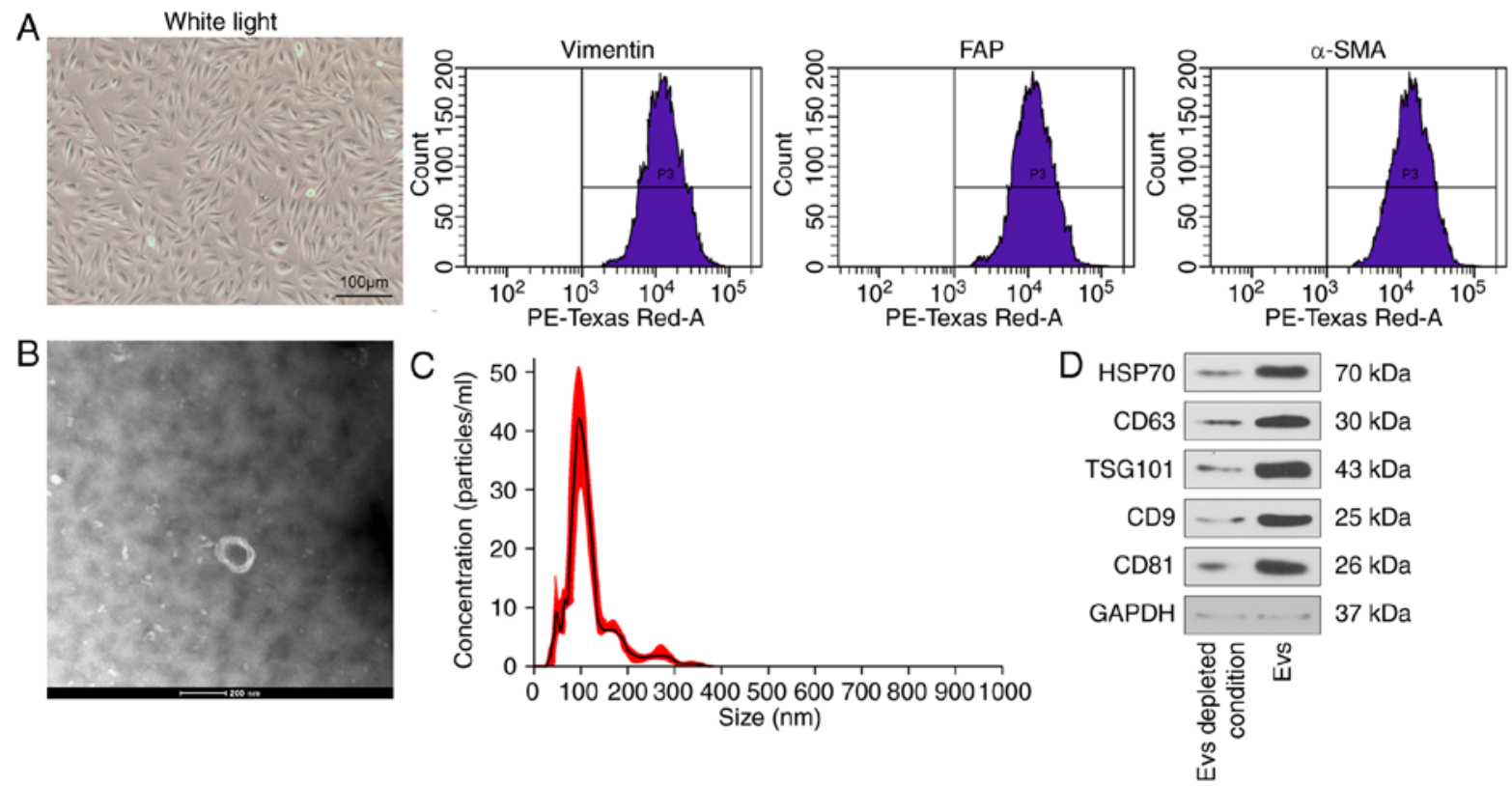

Figure 1. CAFs-derived EVs are successfully isolated and identified. (A) Representative images of CAFs (scale bar=100 $\mu \mathrm{m}$ ) and identification of CAFs using antibodies against vimentin, $\alpha$-SMA, and FAP determined by flow cytometry. (B) Transmission electron microscopy indicating EVs isolated from CAFs-conditioned medium (scale bar=200 nm). (C) Detection of the diameter of CAFs-EVs by nanosight tracking analysis. (D) The bands of EVs markers CD63, HSP70, CD9, CD81 and TSG101 in EV-enriched conditioned medium evaluated by western blot analysis, normalized to GAPDH. CAFs, cancer-associated fibroblasts; EVs, extracellular vesicles; $\alpha$-SMA, $\alpha$-smooth muscle actin; FAP, prolyl endopeptidase FAP; CD63, CD63 antigen; HSP70, heat shock protein 70; CD9, CD9 antigen; CD81, CD81 antigen; TSG101, tumor susceptibility gene 101 protein.

treated with different concentrations of EVs, and it was identified that the optimum concentration was $20 \mu \mathrm{mol} / 1$ (Fig. 2A). The LUSC H520 and SK-MES-1 cell lines were then treated with PBS, $20 \mu \mathrm{mol} / 1 \mathrm{EVs}$ and SDS-treated EVs, respectively. It was identified that, following EVs treatment, the viability of H520 and SK-MES-1 cells increased significantly (Fig. 2B-C), accompanied by significantly induced epithelial-mesenchymal transition (EMT) (Fig. 2D and E), and the number of invasive and migratory H520 and SK-MES-1 cells elevated significantly (Fig. 2F and G). However, following destruction of the membrane structure of EVs using SDS treatment, the promotive roles of EVs were not observed, which indicated that CAFs-derived EVs could promote the malignant biological behavior of LUSC cells.

Expression of miR-369 in LUSC cells is enhanced following treatment with $C A F S-E V s$. Then, a miRNA microarray analysis was used to examine the miRNA expression of H520 cells treated with EVs. It was identified that miR-369 increased significantly following EVs treatment. It was revealed that miR-369 was highly expressed in the EVs extracted from CAFs in the bioinformatics database EvimiR (Fig. 3A and B). The expression levels of miR-369 in H520 and SK-MES-1 cells were then detected by RT-qPCR, which was enhanced by treatment with EVs (Fig. 3C). In addition, according to TCGA database, miR-369 was significantly overexpressed in patients with LUSC (Fig. 3D). Therefore, the miR-369 expression levels were detected in 52 cases of LUSC tissues and the corresponding paratumor tissues. miR-369 was observed to be highly expressed in the LUSC tissues (Fig. 3E). The expression of miR-369 was then downregulated in CAFs and the EVs were extracted for subsequent experiments. RT-qPCR was used to verify the transfection efficiency (Fig. 3F).
Downregulated miR-369 carried by CAFs-EVs partially inhibits the malignancy of LUSC cells. Following the treatment of miR-369 inhibitor in the CAFs-EVs, the malignant biological behaviors of $\mathrm{H} 520$ and SK-MES-1 cells promoted by EVs were significantly decreased (Fig. 4A-F).

miR-369 targets NF1 to activate the MAPK signaling pathway. A large number of mRNAs that were targeted by miR-369 were screened using the StarBase and TargetScan databases. A Kyoto Encyclopedia of Genes and Genomes enrichment analysis was performed for the 471 mRNAs identified, and a total of 61 enriched signaling pathways were identified. The MAPK signaling pathway was screened out, and NF1 was located upstream of the pathway (Fig. 5A-C). It was reported previously that the NF1 mutation could promote or lead to the occurrence of lung adenocarcinomas (16). Therefore, a dual luciferase assay was used to validate the binding connection between miR-369 and NF1 (Fig. 5D). Subsequently, the expression of NF1 in the LUSC tissues and the corresponding paratumor tissues was examined, and the NF1 mRNA expression in the LUSC tissues was significantly decreased (Fig. 5E). Then, the expression of NF1, MEK1 and ERK1/2, and the extent of MEK1 and ERK1/2 phosphorylation in cells, were detected by ELISAs. It was identified that CAFs-EVs treatment could inhibit NF1 protein expression and increase the extent of MEK1 and ERK1/2 phosphorylation. Then, miR-369 expression in EVs was inhibited using transfection, and it was observed that that the expression of NF1 was significantly increased, while the MEK1 and ERK1/2 phosphorylation levels significantly decreased (Fig. 5F).

CAFs-EVs treatment promotes the growth and metastasis of LUSC in vivo. With the aim of verifying the role of 

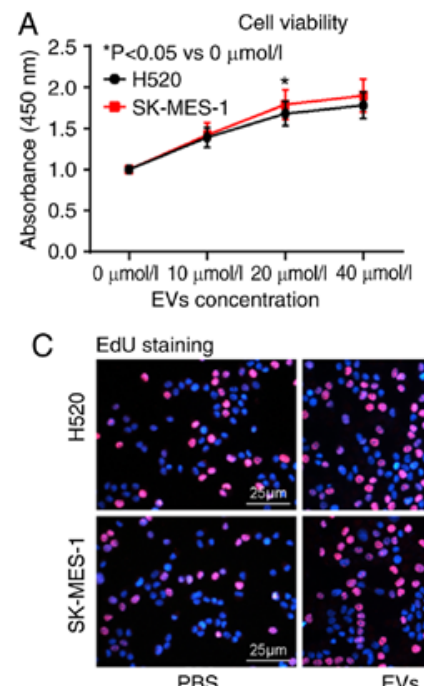

PBS

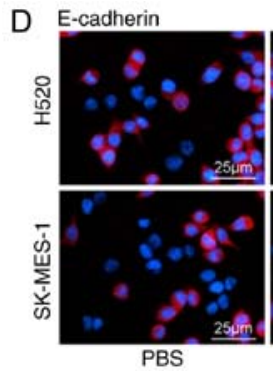

E

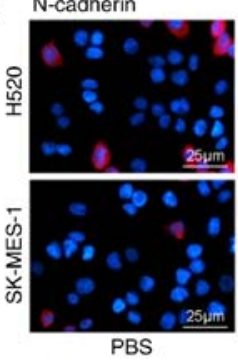

F

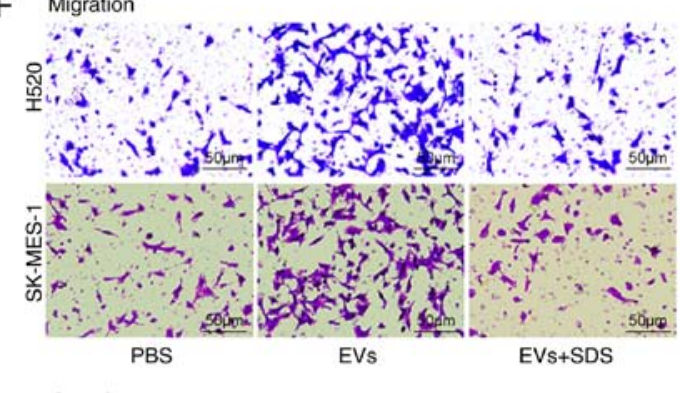

$G$
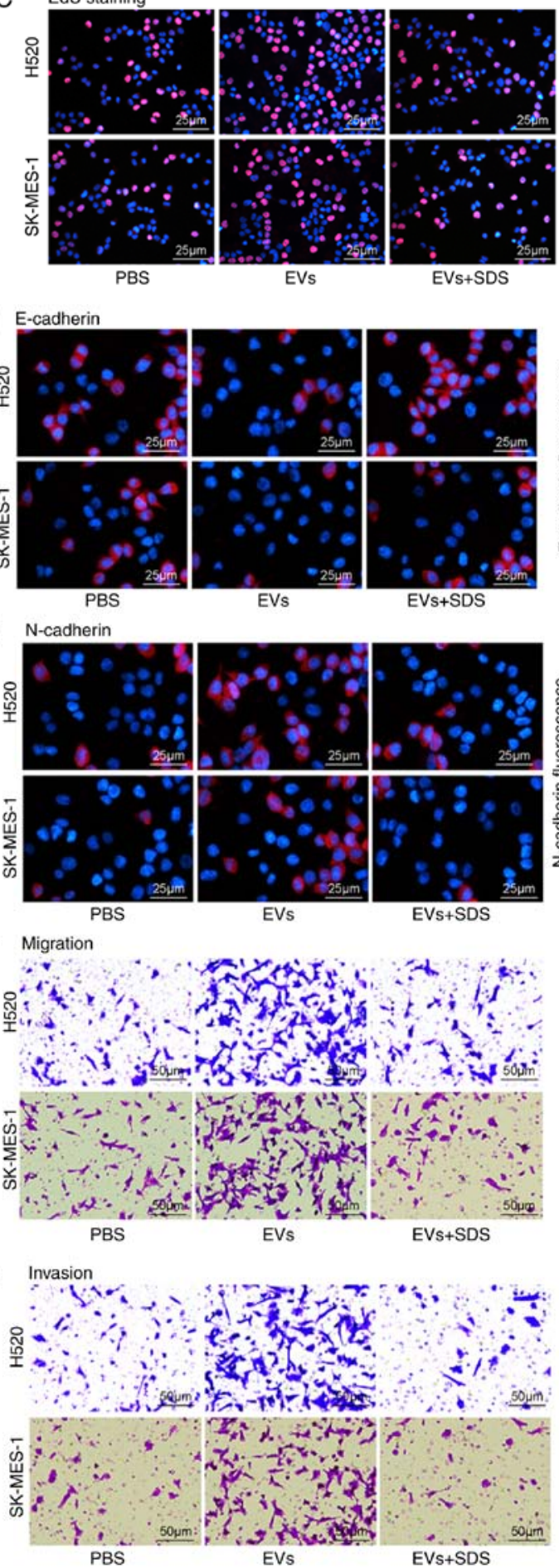

EVs+SDS
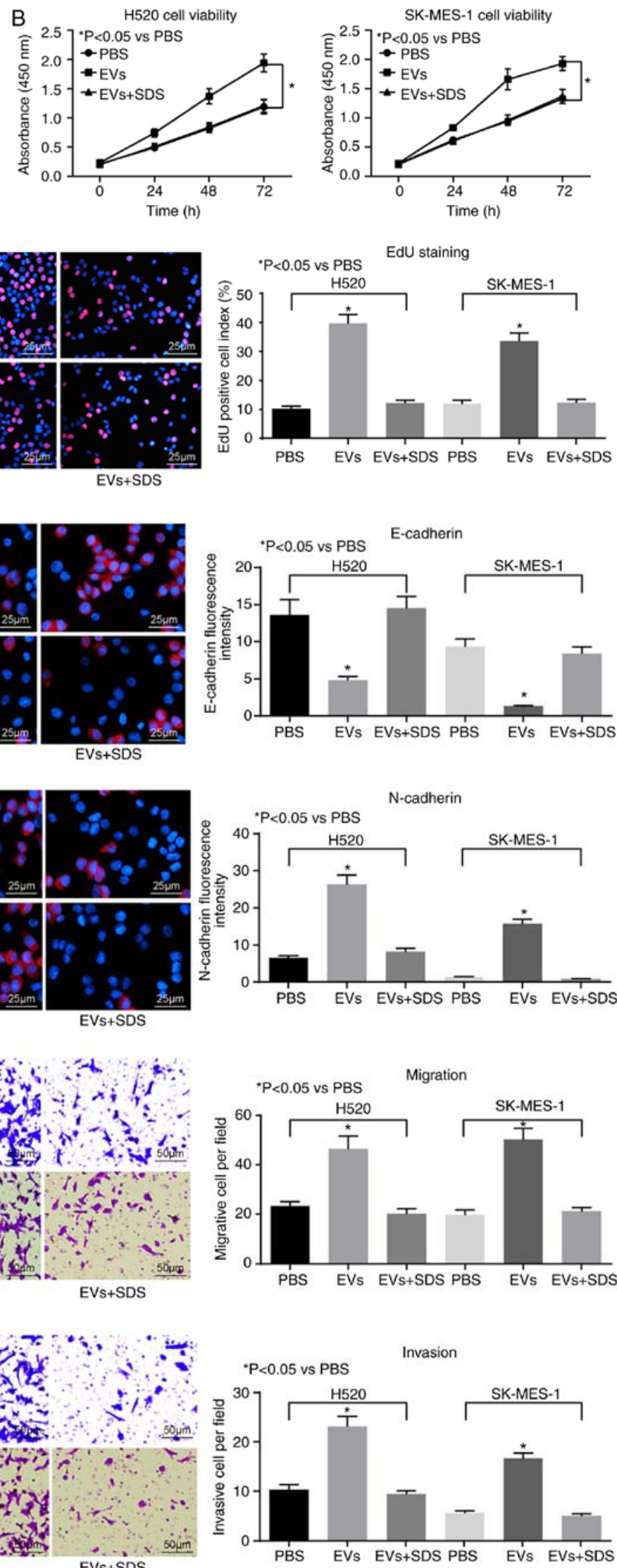

Figure 2. CAFs-derived EVs promote lung squamous cell carcinoma cells malignancy. (A) The concentration of EVs determined by Cell Counting Kit-8 assay. (B) The viability of H520 cells assessed by EdU assay. (C) The viability of SK-MES-1 cells assessed by EdU assay. (D) Immunofluorescence staining of EMT marker E-cadherin. (E) Immunofluorescence staining of EMT marker N-cadherin. (F) Cell migration ability measured by Transwell assay. (G) Cell invasion ability measured by Transwell assay. Data are expressed as mean \pm SD. In panel A and B, a two-way ANOVA and Tukey's multiple comparisons test was used to determine statistical significance, while in panel $\mathrm{C}$ to $\mathrm{G}$, a one-way ANOVA was used. ${ }^{*} \mathrm{P}<0.05$ vs. PBS treatment. CAFs, cancer-associated fibroblasts; EVs, extracellular vesicles; EdU, 5-ethynyl-2'-deoxyuridine; EMT, epithelial-mesenchymal transition; E-cadherin, epithelial cadherin; N-cadherin, neural cadherin; ANOVA, analysis of variance. 


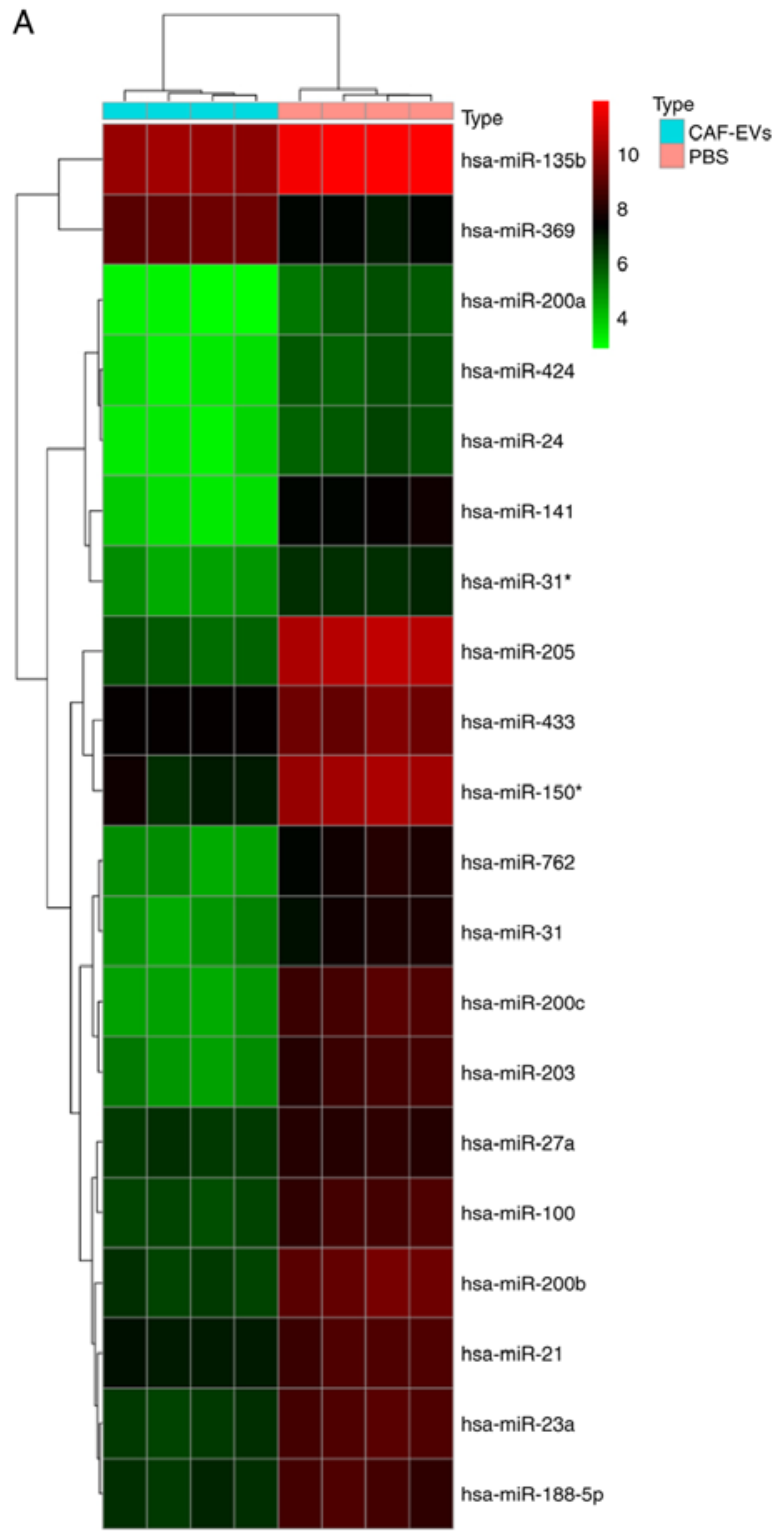

D hsa-miR-369-3p with 475 cancer and 38 normal samples in LUSC

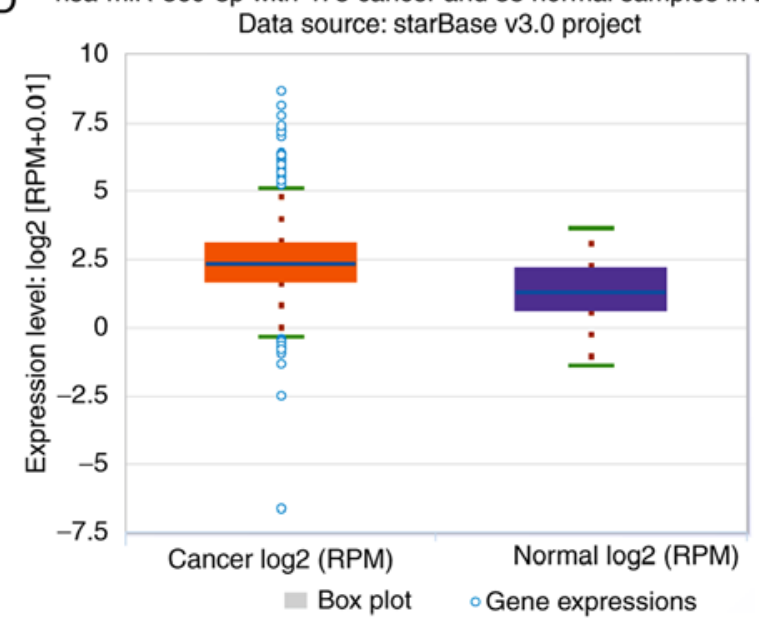

B hsa-miR-369-3p Expression of extracellular vesicles - Evs source Evs cancer

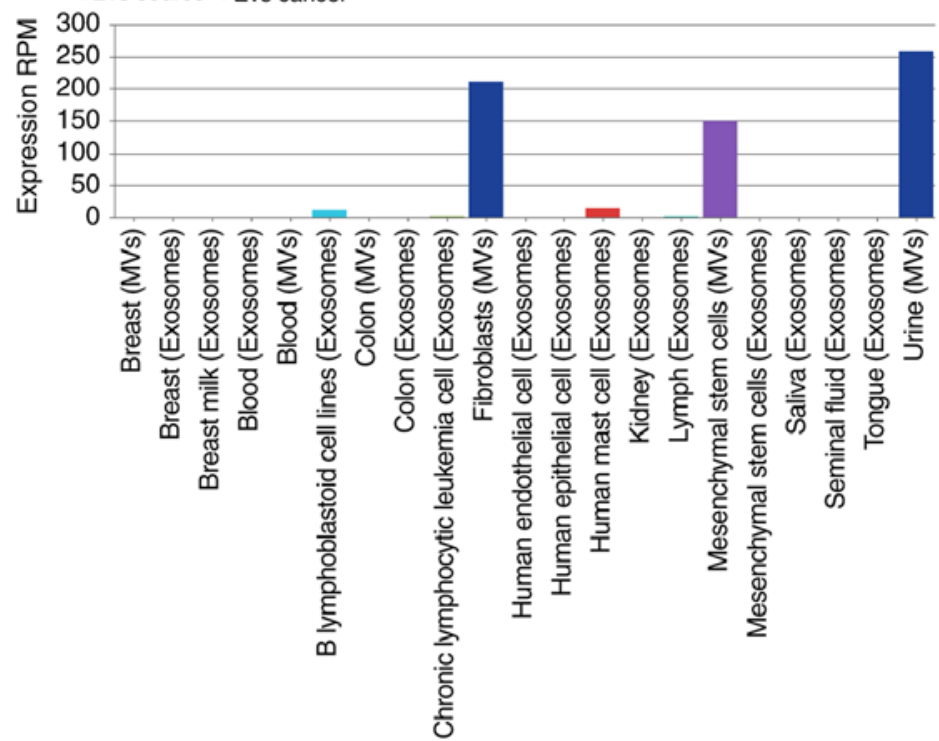

C

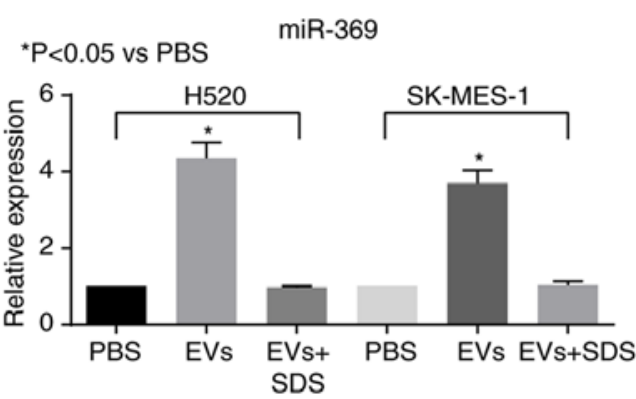

$E$

miR-369

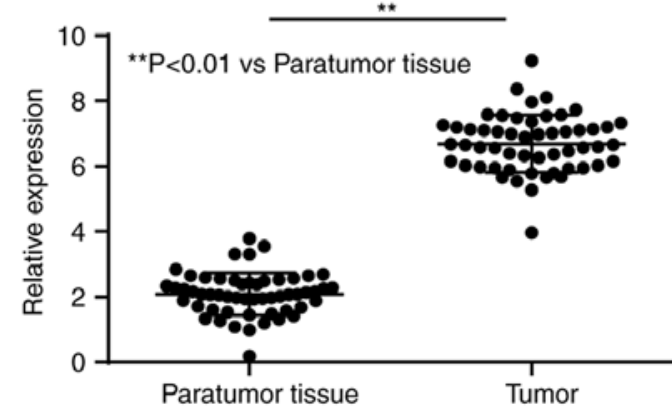

$\mathrm{F}$

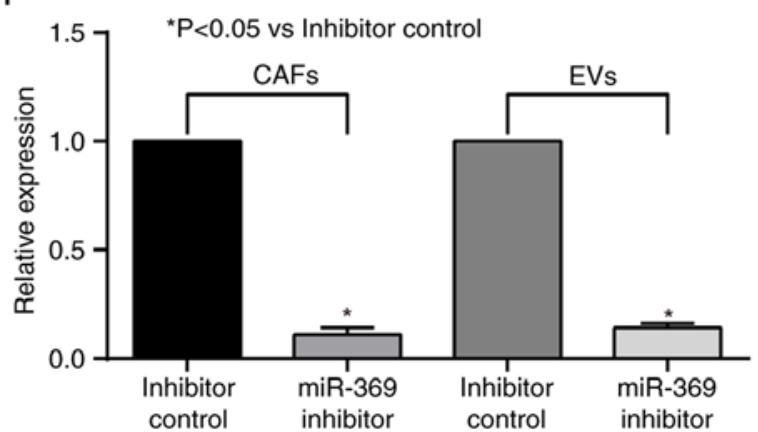

Figure 3 CAFs-derived EVs upregulate miR-369 expression in LUSC cells. (A) Dysregulated miRNAs following EVs treatment predicted using miRCURY LNA $^{\mathrm{TM}}$ Universal RT microRNA PCR Human panel. (B) The miR-369 expression in all tumor types. (C) miR-369 expression in H520 and SK-MES-1 cells following EVs treatment measured by RT-qPCR. (D) The miR-369 expression in patients with LUSC and normal individuals in The Cancer Genome Atlas datasets. (E) The miR-369 expression in 52 LUSC tumor tissues and paracancerous tissues measured by RT-qPCR. (F) miR-369 expression in CAFs and its EVs treated with miR-369 inhibitor or inhibitor control. Data are expressed as mean \pm SD. In panels C and F, one-way analysis of variance and Tukey's multiple comparisons test was used to determine statistical significance. ${ }^{*} \mathrm{P}<0.05$ compared with inhibitor control. ${ }^{* *} \mathrm{P}<0.01$ compared with paratumor tissues. CAFs, cancer-associated fibroblasts; EVs, extracellular vesicles; miR, microRNA; LUSC, lung squamous cell carcinoma; RT-qPCR, reverse transcription-quantitative PCR. 
B

EdU staining

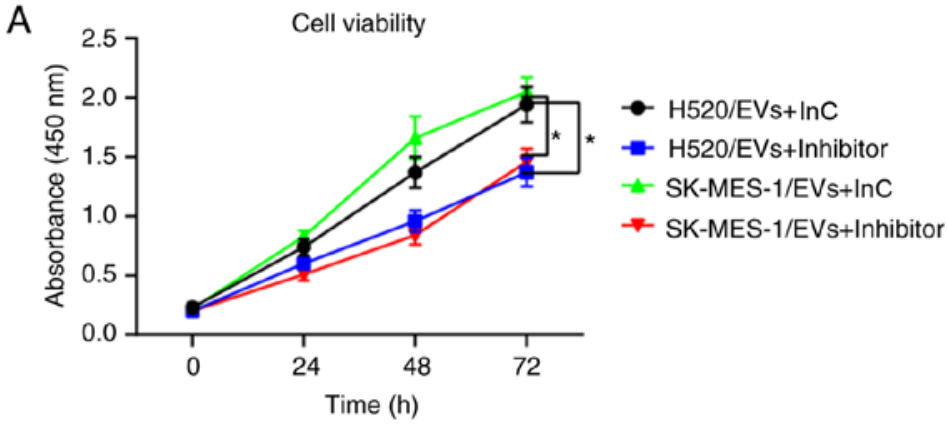



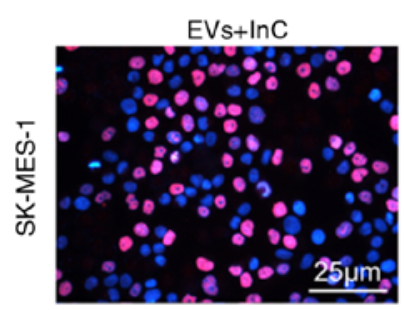

C
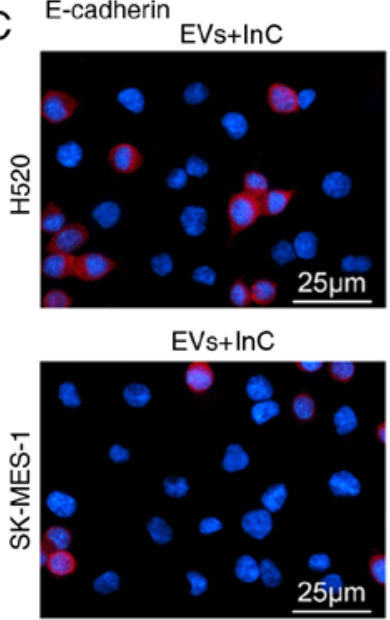

D $\mathrm{N}$-cadherin $\mathrm{EVs}+\ln \mathrm{C}$

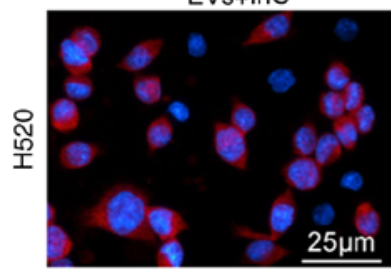

$E V s+\ln C$

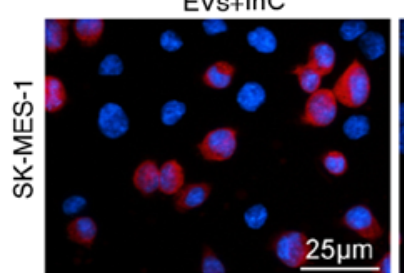

EVs+Inhibitor
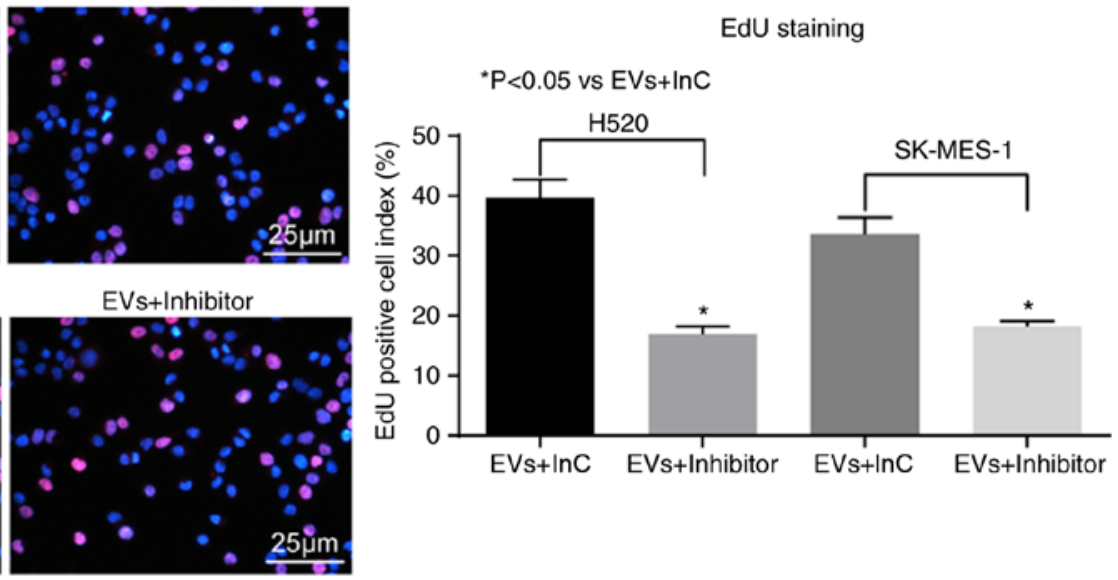

EdU staining



EVs+Inhibitor
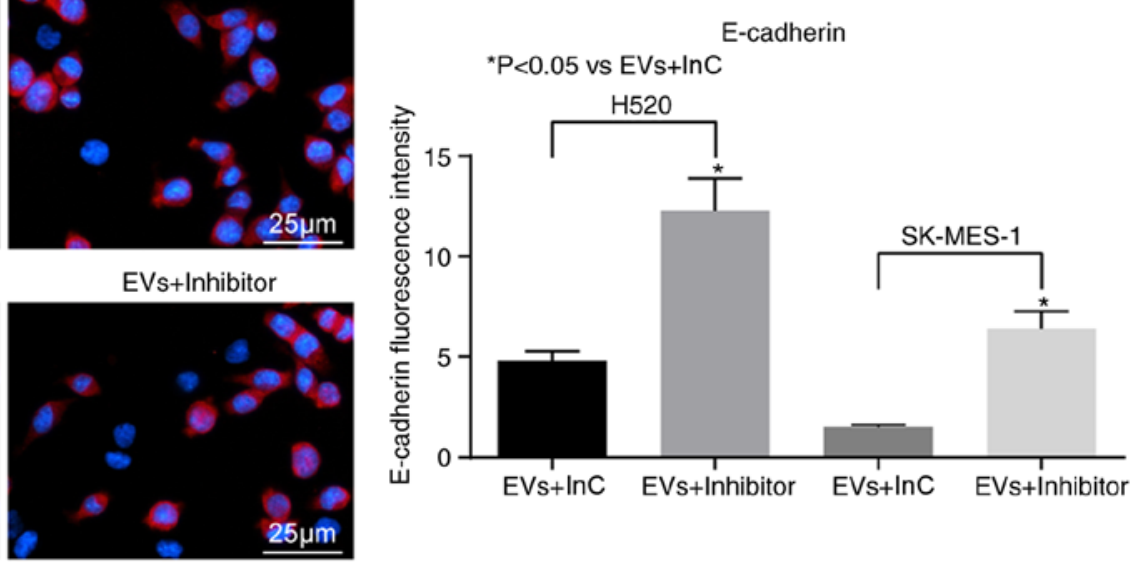

$\mathrm{N}$-cadherin

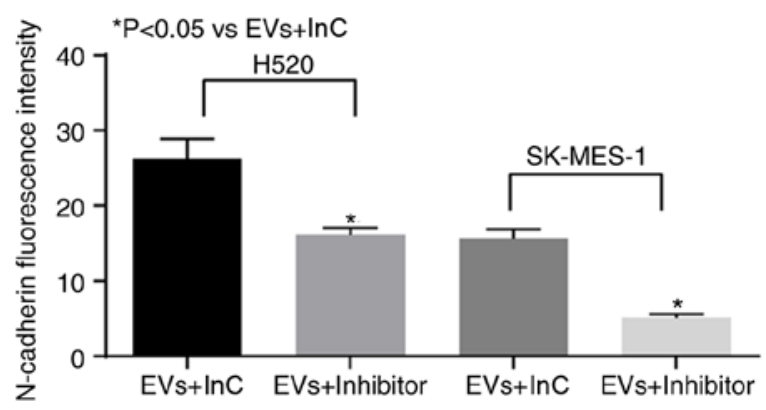

Figure 4. miR-369 inhibition in CAFs-derived EVs partially reverses the effects of EVs on LUSC cells malignancy. (A) The concentration of EVs determined by Cell Counting Kit-8 assay. (B) The viability of H520 and SK-MES-1 cells assessed by EdU assay. (C) Immunofluorescence staining of EMT marker E-cadherin in H520 and SK-MES-1 cells. (D) Immunofluorescence staining of EMT marker N-cadherin in H520 and SK-MES-1 cells. 



F Invasion
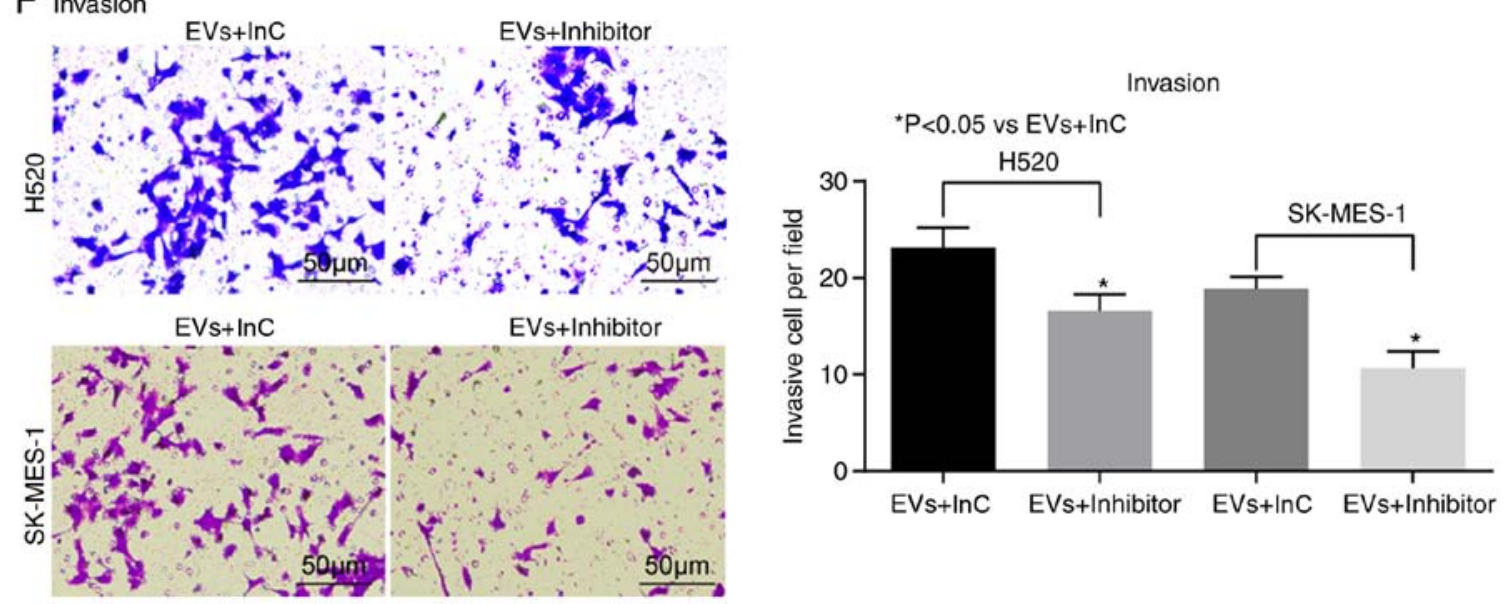

Figure 4 (Continued). (E) H520 and SK-MES-1 cell migration ability measured by Transwell assay. (F) H520 and SK-MES-1 cell invasion ability measured by Transwell assay. Data are expressed as mean \pm SD. In panel A, two-way ANOVA and Tukey's multiple comparisons test was used to determine statistical significance. In panel B to F, one -way ANOVA was used. "P<0.05 vs. EVs + InC treatment. miR, microRNA; CAFs, cancer-associated fibroblasts; EVs, extracellular vesicles; EdU, 5-ethynyl-2'-deoxyuridine; EMT, epithelial-mesenchymal transition; E-cadherin, epithelial cadherin; N-cadherin, neural cadherin; InC, Inhibitor control; ANOVA, analysis of variance.

CAFs-derived EVs on the development and metastasis of LUSC in vivo, in vivo tumorigenesis and in vivo metastasis models were established in nude mice. In the in vivo tumorigenesis experiment, it was identified that EVs treatment could promote the tumor volume and weight in vivo and increase the $\mathrm{Ki}-67$-positive cell rate of transplanted tumors (Fig. 6A-C). Furthermore, in the in vivo metastasis model, it was identified that the number of liver metastases and lung metastases increased significantly following EVs treatment (Fig. 6D).

\section{Discussion}

Cancer is closely associated with fibroblast activity at all stages of disease progression, including metastasis, and CAFs may responsible for the generation of various tumor components (27). The primary result of the present study is the marked upregulation of miR-369 in LUSC tissues compared with the paratumor tissues. In addition, miR-369 inhibition in the CAF-derived EVs decreased LUSC cell proliferation, migration and invasion in vitro, and lung and liver metastases in vivo. Additionally, it was demonstrated that miR-369 in
CAF-derived EVs activated the MAPK signaling pathway by directly binding to NF1. These results suggest that the dysregulation of miR-369 may serve a paramount role in accelerating tumorigenesis and metastases in LUSC.

According to EvimiR, an important miRNA database, miR-369 was significantly upregulated in CAF-derived EVs. EVs have been demonstrated to carry nucleic acids including miRNA, mRNA and non-coding RNAs (28). Baffa et al (29) observed that miR-369-3p was overexpressed in metastatic NSCLC tissues. The results of the present study demonstrated an association between the upregulation of miR-369 expression in CAF-EVs and LUSC cell migration, invasion and tumor progression, and suggested that the NF1-mediated MAPK signaling pathway may be involved. By using the publicly available databases StarBase and TargetScan, a conserved binding site of miR-369 on the 3'UTR of NF1 gene was identified, which was further confirmed by luciferase reporter assays. Therefore, miR-369 may act as a positive regulator of LUSC cell migration and invasion via specific down-regulation of NF1. NF1 was reported to be highly expressed in NSCLC tissues and A549 and HCC823 cells compared with the controls (30). Aberrations in NF1 contribute to the dysregulation of the 
A

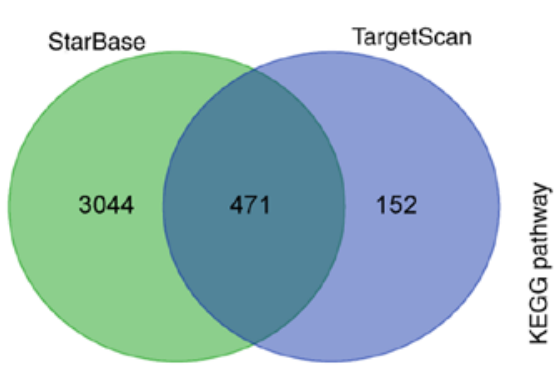

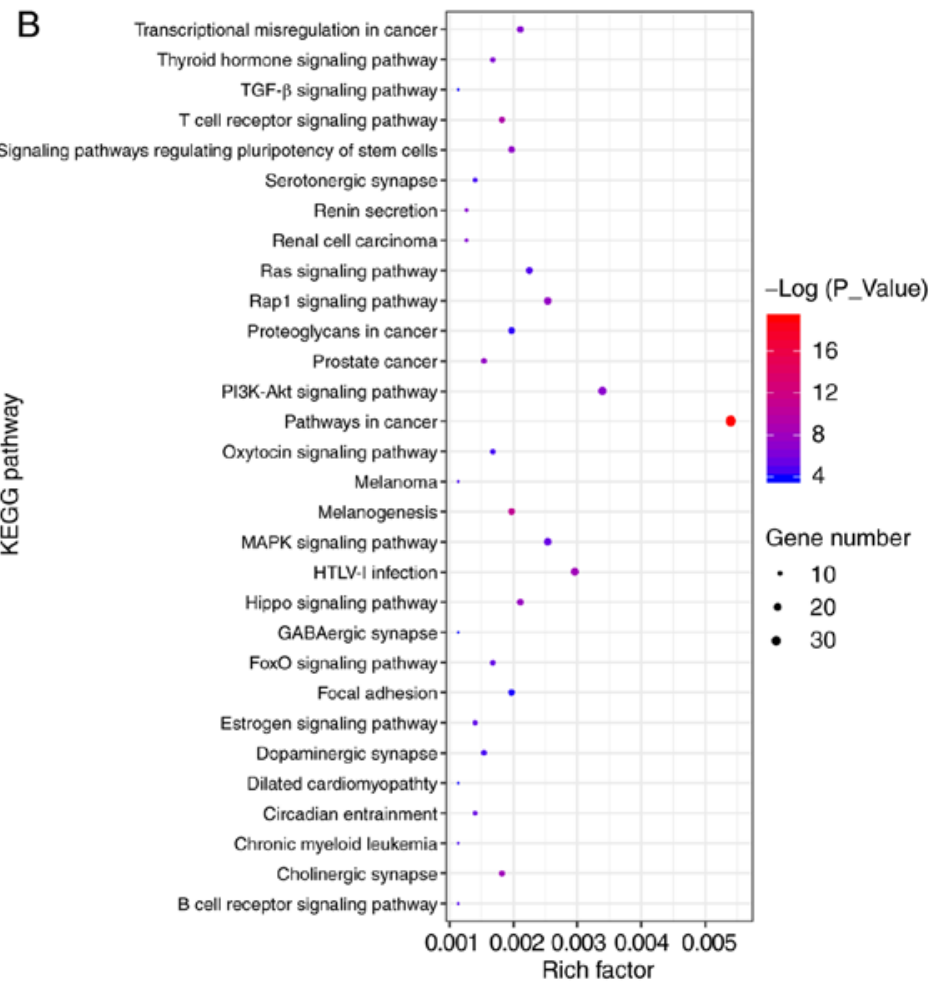

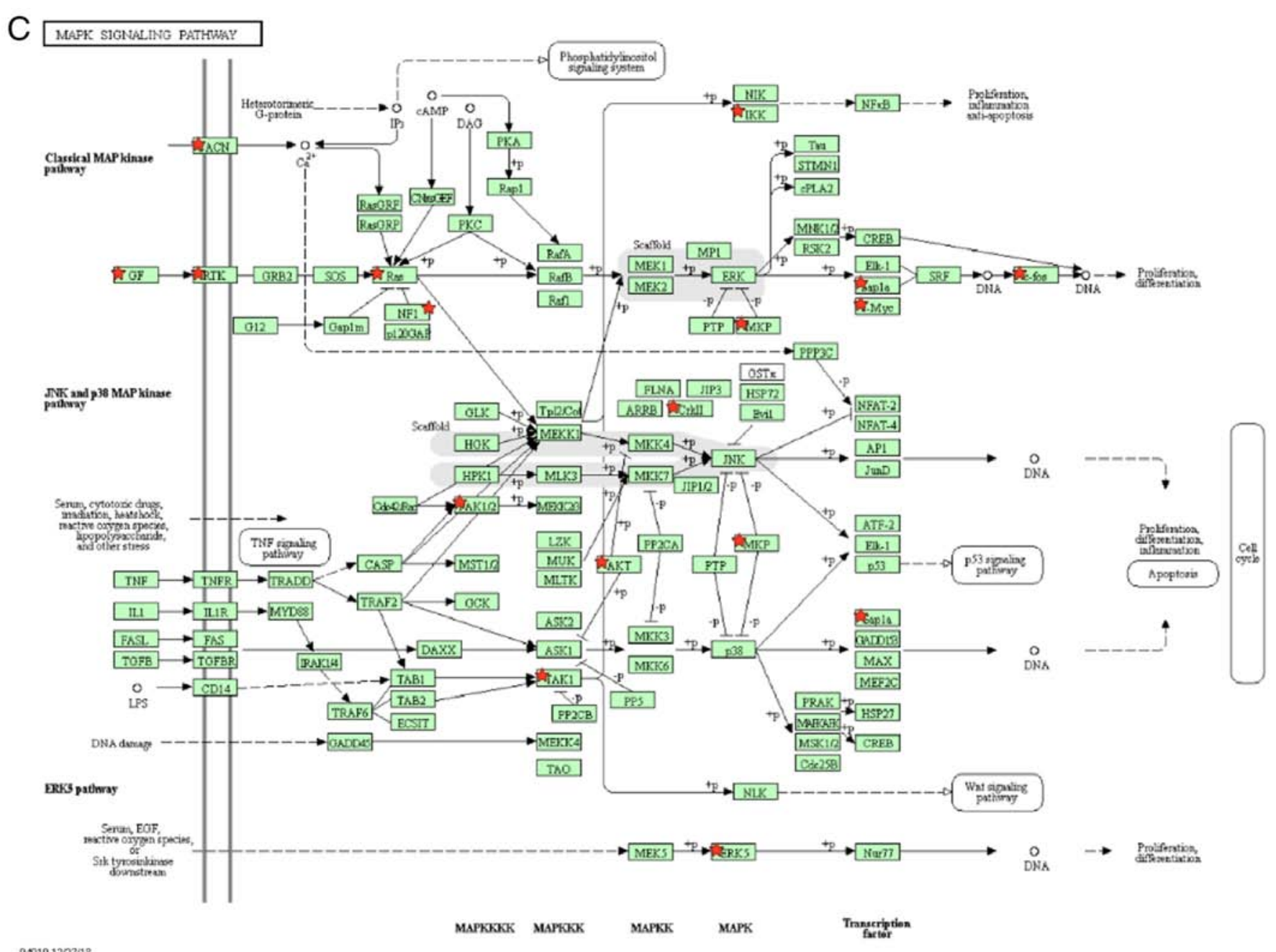

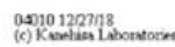

Figure 5. miR-369 activates the MAPK signaling pathway by targeting the tumor suppressor NF1. (A) The mRNA targets of miR-369 were predicted by TargetScan and StarBase, and 471 mRNA was screened out. (B) Signaling pathways enriched by targeting mRNAs of miR-369 analyzed by KEGG. (C) The MAPK signaling pathway enriched by targeting mRNAs of miR-369 analyzed by KEGG. 




$\mathrm{E}$

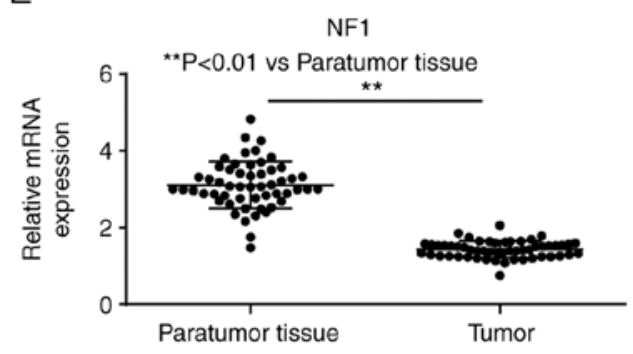

$\mathrm{F}$
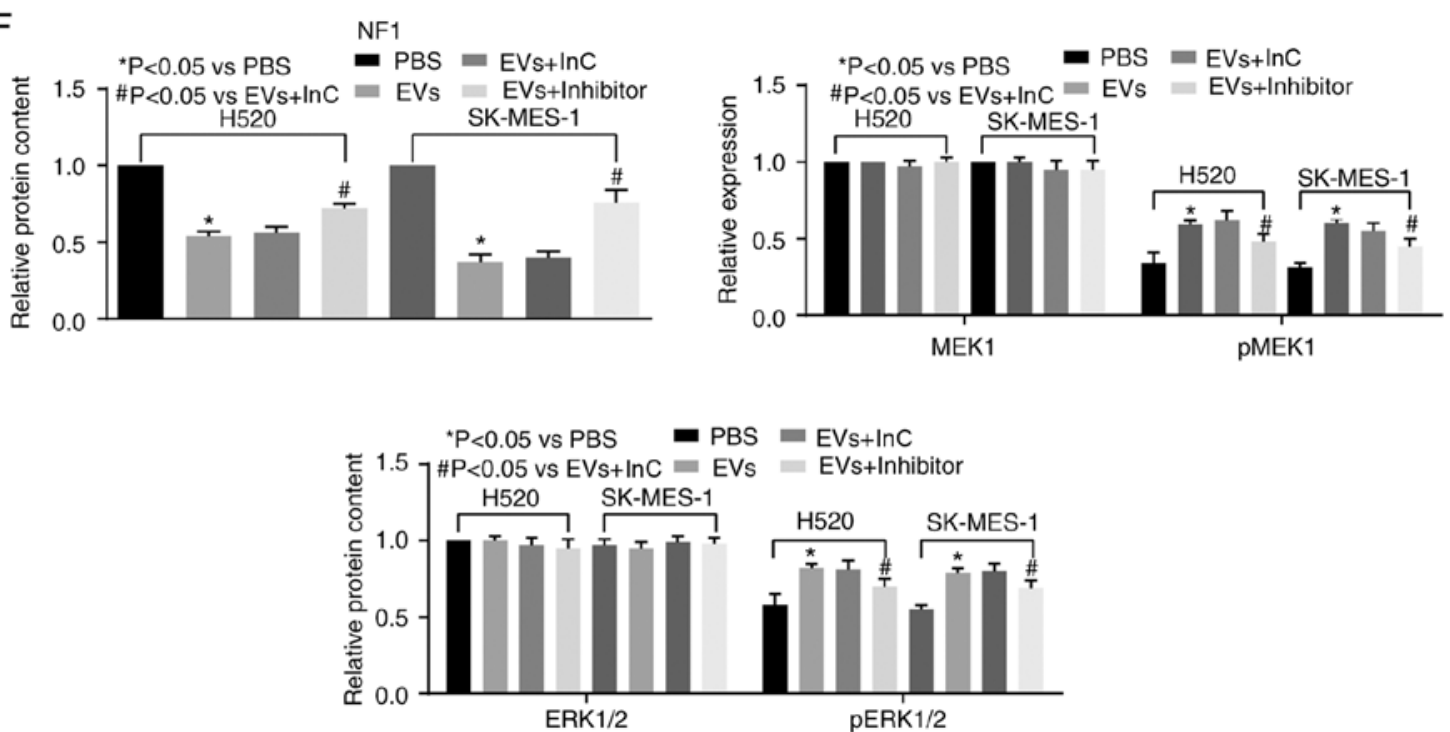

Figure 5 (Continued). (D) Predicted miRNA binding sites within the 3'-UTR of NF1 mRNA by StarBase. The pairing between miR-369 and the putative binding sites in the 3'-UTR of APC mRNA are shown. Data were analyzed using a one-way ANOVA followed by Tukey's post hoc test. (E) The NF1 expression levels in 52 LUSC tumor tissues and paratumor tissues were measured by reverse transcription-quantitative PCR. Data were analyzed using a paired t-test. (F) The protein expression levels of NF1, MEK1, pMEK1, ERK1/2 and pERK1/2 were determined by ELISA. Data are expressed as mean \pm SD. Data were analyzed using a one-way ANOVA followed by Tukey's post hoc test. ${ }^{*} \mathrm{P}<0.05$ vs. PBS treatment. ${ }^{~} \mathrm{P}<0.05$ vs. EVs + InC treatment. ${ }^{* *} \mathrm{P}<0.01$ compared with paratumor tissues. miR, microRNA; NF1, neurofibromin 1; UTR, untranslated region; MEK1, mitogen-activated protein kinase/ERK kinase 1; ERK1/2, extracellular signal-regulated kinase 1/2; p, phosphorylated; InC, inhibitor control; KEGG, Kyoto Encyclopedia of Genes and Genomes.

RAS/MAPK signaling pathway, culminating in disfunction of cell growth and proliferation (31).

Furthermore, CAFs-EVs promoted the migration, invasion and EMT of LUSC cells in vitro in the present study. Exosomes extracted from the human sera of samples from late-stage lung cancer enhanced vimentin expression and stimulated the migration, invasion and EMT of human bronchial epithelial cells (32). In addition, CAFs induced EMT in lung cancer cells via exosomes in a zinc finger protein SNAI1-dependent manner (33). Exosomes containing miR-499a released from a highly metastatic cell line increased cell proliferation, migration and EMT in lung adenocarcinoma samples, and the trends were reversed by the suppression of miR-499a-5p (34). Vimentin has been confirmed to participate in cancer tumorigenesis, EMT and cellular metastatic properties (32). Notably, CAFs-EVs exhibited stimulatory effects on the growth of the H520 and SK-MES-1 cell lines in vivo, suggesting that the inhibition of CAFs-EVs may be a potential therapeutic strategy in LUSC. Consistent with the data from the present study, Verset et al (35) provided quantitative data demonstrating the increased expression of CAFs in the cancer-associated stroma in rectal cancer. Exosomes antagonized the protective effect of mesothelial cells and facilitated the metastasis of tumor cells, particularly in fluid ascites, implying that exosomes may induce the transformation of mesothelial cells into CAFs to promote metastasis (36). Similar to the results of the present study, melanoma cells were demonstrated to control the creation of the dermal tumor niche by inducing EVs overexpressing miR-211, which directly interacted with the insulin-like growth factor 2 receptor, contributing to the potentiation of the MAPK signaling pathway that encourages melanoma cell growth (37). In addition, gastric cancer cell-induced exosomes promoted the proliferation and migration of pericytes and enhanced the CAFs marker expression in pericytes, during which the MEK/ERK pathway was activated by tumor-derived exosomes (38). The present study demonstrated that NF1 was expressed at decreased levels in the LUSC tissues compared with the matched paratumor tissues, while the treatment of EVs with the miR-369 inhibitor significantly enriched 

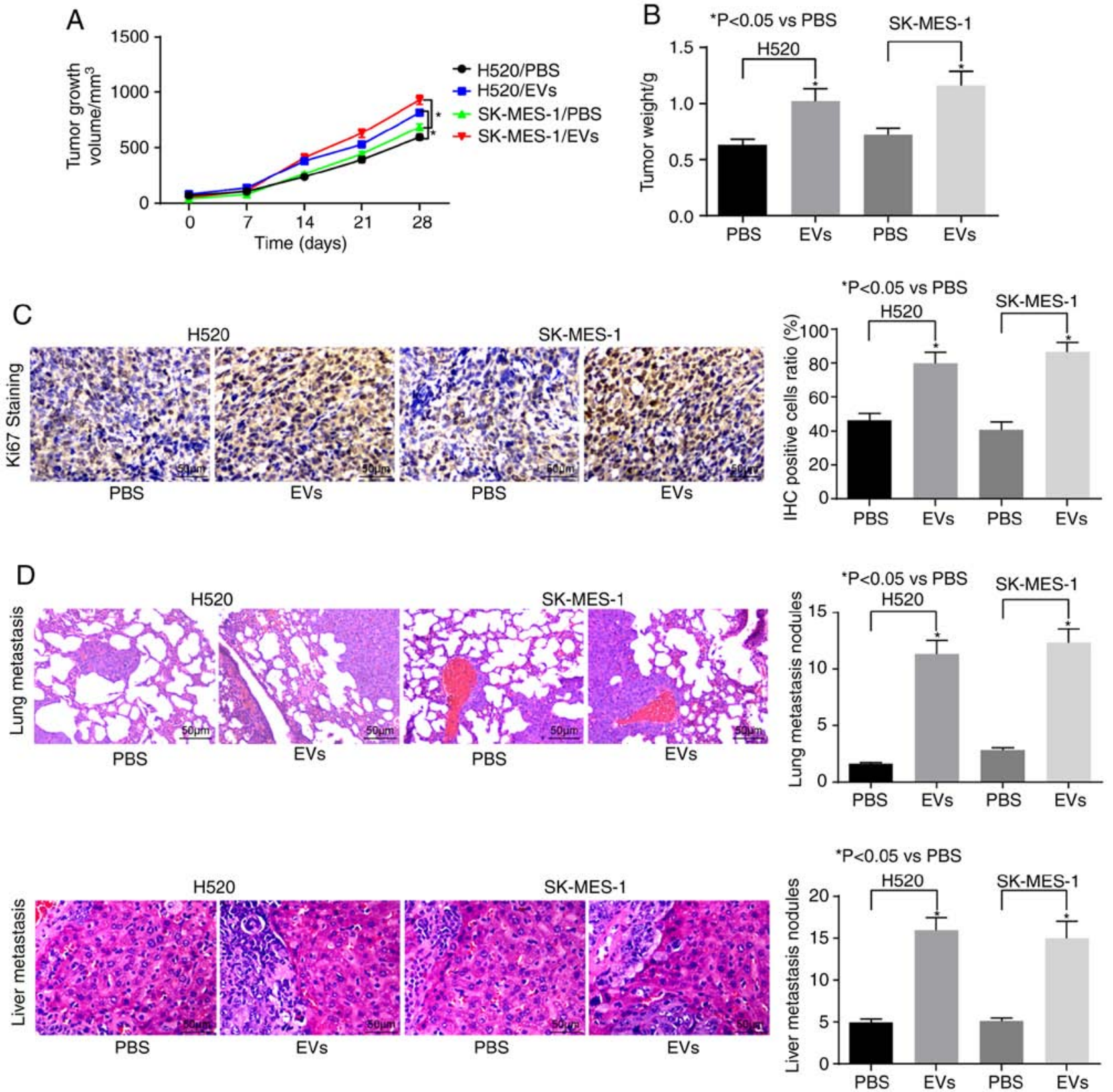

Figure 6. Cancer-associated fibroblast-derived EVs accelerate the progression and metastasis of lung squamous cell carcinoma in vivo. Nude mice were subcutaneously injected with H520 and SK-MES-1 cells diluted with PBS or EVs. (A) Tumor volume of nude mice on days 0, 7, 14, 21 and 28 post-injection. (B) Tumors were excised and weighed 28 days after implantation. Mean tumor weights in each group are shown in the histogram. (C) Representative images of Ki-67-positive tumor cells and quantification of immunostaining results. (D) Hematoxylin \& eosin staining of lung and liver metastases in nude mice injected with H520 and SK-MES-1 cells through the tail vein. The numbers of metastasis were determined at 45 days after H520 and SK-MES-1 cells injection. $n=10$. One-way or two-way analysis of variance and Tukey's multiple comparison test was used to determine statistical significance, ${ }^{*} \mathrm{P}<0.05$ vs. H520 or SK-MES-1 cells treated with PBS. EVs, extracellular vesicles; Ki-67, proliferation marker protein Ki-67; IHC, immunohistochemistry.

the expression of NF1 and decreased the extent of MEK1 and ERK1/2 phosphorylation.

In summary, the present study identified miR-369 as a novel potential oncogene in LUSC that acts by inducing EMT and the metastasis process. It was observed that the silencing of miR-369 in CAF-EVs significantly inhibited tumor growth and metastasis in vivo, suggesting that miR-369 inhibition may be a capable therapeutic target for LUSC through CAF-EVs. Further studies should examine the association between EVs and LUSC cells further, which may optimize the current therapeutic strategies for LUSC.

\section{Acknowledgments}

The results published here are in whole or part based upon data generated by the TCGA Research Network: https://www. cancer.gov/tcga.

\section{Funding}

This study was supported by the University Level Scientific Research Project of Yan'an University (grant nos. YDQ2019-35, D2017197 and YDJGY19-02). 


\section{Availability of data and materials}

All the data generated or analyzed during this study are included in this published article.

\section{Authors' contributions}

LG contributed to the conception and design of the study. BL contributed to the experiments and clinical studies. JY contributed to the data and statistical analyses. JS was involved in manuscript preparation and acquisition of experimental data. $\mathrm{JJ}$ and MM contributed to analysis and interpretation of data, and reviewed the manuscript. All authors are responsible for the integrity of the data and have read and approved the final manuscript.

\section{Ethics approval and consent to participate}

All experiments were completed following the protocol that was approved by the Animal Care and Use Committee of Affiliated Hospital of Yan'an University. All efforts were made to minimize the number of animals applied in the experiments and their suffering. All human tissue donors provided written informed consent prior to tissue donation. The study protocol was approved by the Institutional Review Board of Affiliated Hospital of Yan'an University.

\section{Patient consent for publication}

Not applicable.

\section{Competing interests}

The authors declare that they have no competing interests.

\section{References}

1. Siegel RL, Miller KD and Jemal A: Cancer statistics, 2020. CA Cancer J Clin 70: 7-30, 2020.

2. Torre LA, Siegel RL and Jemal A: Lung Cancer Statistics. Ady Exp Med Biol 893: 1-19, 2016.

3. Xu F, Zhang H, Chen J, Lin L and Chen Y: Immune signature of $\mathrm{T}$ follicular helper cells predicts clinical prognostic and therapeutic impact in lung squamous cell carcinoma. Int Immunopharmacol 81: 105932, 2020.

4. Shi Y, Li Y, Yan C, Su H and Ying K: Identification of key genes and evaluation of clinical outcomes in lung squamous cell carcinoma using integrated bioinformatics analysis. Oncol Lett 18: 5859-5870, 2019

5. Erez N, Truitt M, Olson P, Arron ST and Hanahan D: Cancer-associated fibroblasts are activated in incipient neoplasia to orchestrate tumor-promoting inflammation in an NF-kappaB-dependent manner. Cancer Cell 17: 135-147, 2010.

6. Hanley CJ, Mellone M, Ford K, Thirdborough SM, Mellows T, Frampton SJ, Smith DM, Harden E, Szyndralewiez C, Bullock M, et al: Targeting the Myofibroblastic Cancer-Associated Fibroblast Phenotype Through Inhibition of NOX4. J Natl Cancer Inst 110: 2018.

7. Raposo G and Stoorvogel W: Extracellular vesicles: Exosomes, microvesicles, and friends. J Cell Biol 200: 373-383, 2013.

8. Yang X, Li Y, Zou L and Zhu Z: Role of exosomes in crosstalk between cancer-associated fibroblasts and cancer cells. Front Oncol 9: 356, 2019.

9. Sun LP, Xu K, Cui J, Yuan DY, Zou B, Li J, Liu JL, Li KY, Meng Z and Zhang B: Cancer-associated fibroblast-derived exosomal miR3825p promotes the migration and invasion of oral squamous cell carcinoma. Oncol Rep 42: 1319-1328, 2019.
10. Hao GJ, Ding YH, Wen H, Li XF, Zhang W, Su HY, Liu DM and Xie NL: Attenuation of deregulated miR-369-3p expression sensitizes non-small cell lung cancer cells to cisplatin via modulation of the nucleotide sugar transporter SLC35F5. Biochem Biophys Res Commun 488: 501-508, 2017.

11. Li JH, Liu S, Zhou H, Qu LH and Yang JH: starBase v2.0: Decoding miRNA-ceRNA, miRNA-ncRNA and protein-RNA interaction networks from large-scale CLIP-Seq data. Nucleic Acids Res 42 (Database issue): D92-D97, 2014.

12. Agarwal V, Bell GW, Nam JW and Bartel DP: Predicting effective microRNA target sites in mammalian mRNAs. Elife 4: 2015.

13. Tlemsani C, Pecuchet N, Gruber A, Laurendeau I, Danel C, Riquet M, Le Pimpec-Barthes F, Fabre E, Mansuet-Lupo A, Damotte D, et al: NF1 mutations identify molecular and clinical subtypes of lung adenocarcinomas. Cancer Med 8: 4330-4337, 2019.

14. Brems H, Park C, Maertens O, Pemov A, Messiaen L, Upadhyaya M, Claes K, Beert E, Peeters K, Mautner V, et al: Glomus tumors in neurofibromatosis type 1: Genetic, functional, and clinical evidence of a novel association. Cancer Res 69: 7393-7401, 2009.

15. Stites EC, Trampont PC, Haney LB, Walk SF and Ravichandran KS: Cooperation between Noncanonical Ras Network Mutations. Cell Rep 10: 307-316, 2015.

16. Wang X, Min S, Liu H, Wu N, Liu X, Wang T, Li W, Shen Y, Wang H, Qian Z, et al: Nf1 loss promotes Kras-driven lung adenocarcinoma and results in Psat1-mediated glutamate dependence. EMBO Mol Med 11: pii: e9856, 2019.

17. Redig AJ, Capelletti M, Dahlberg SE, Sholl LM, Mach S, Fontes C, Shi Y, Chalasani P and Jänne PA: Clinical and molecular characteristics of NF1-mutant lung cancer. Clin Cancer Res 22: 3148-3156, 2016.

18. Modak JM, Roy-O'Reilly M, Zhu L, Staff I and McCullough LD: Differential MicroRibonucleic acid expression in cardioembolic stroke. J Stroke Cerebrovasc Dis 28: 121-124, 2019.

19. Kanehisa M, Sato Y, Furumichi M, Morishima K and Tanabe M: New approach for understanding genome variations in KEGG. Nucleic Acids Res 47: D590-D595, 2019.

20. Livak KJ and Schmittgen TD: Analysis of relative gene expression data using real-time quantitative PCR and the 2(-Delta Delta C(T)) method. Methods 25: 402-408, 2001.

21. Zhang Y, Guo L, Li Y, Feng GH, Teng F, Li W and Zhou Q: MicroRNA-494 promotes cancer progression and targets adenomatous polyposis coli in colorectal cancer. Mol Cancer 17: 1, 2018.

22. Ji Y, Han Z, Shao L and Zhao Y: Evaluation of in vivo antitumor effects of low-frequency ultrasound-mediated miRNA-133a microbubble delivery in breast cancer. Cancer Med 5: 2534-2543, 2016.

23. Wei S, Peng L, Yang J, Sang H, Jin D, Li X, Chen M, Zhang W, Dang $Y$ and Zhang G: Exosomal transfer of miR-15b-3p enhances tumorigenesis and malignant transformation through the DYNLT1/Caspase-3/Caspase-9 signaling pathway in gastric cancer. J Exp Clin Cancer Res 39: 32, 2020.

24. Huang XY, Huang ZL, Huang J, Xu B, Huang XY, Xu YH, Zhou J and Tang ZY: Exosomal circRNA-100338 promotes hepatocellular carcinoma metastasis via enhancing invasiveness and angiogenesis. J Exp Clin Cancer Res 39: 20, 2020.

25. Wu Y, Liu H, Shi X, Yao Y, Yang W and Song Y: The long non-coding RNA HNF1A-AS1 regulates proliferation and metastasis in lung adenocarcinoma. Oncotarget 6: 9160-9172, 2015.

26. Thery C, Witwer KW, Aikawa E, Alcaraz MJ, Anderson JD, Andriantsitohaina R, Antoniou A, Arab T, Archer F, Atkin-Smith GK, et al: Minimal information for studies of extracellular vesicles 2018 (MISEV2018): A position statement of the International Society for Extracellular Vesicles and update of the MISEV2014 guidelines. J Extracell Vesicles 7: 1535750, 2018.

27. Kalluri R: The biology and function of fibroblasts in cancer. Nat Rev Cancer 16: 582-598, 2016.

28. Ab Razak NS, Ab Mutalib NS, Mohtar MA and Abu N: Impact of chemotherapy on extracellular vesicles: Understanding the chemo-EVs. Front Oncol 9: 1113, 2019.

29. Baffa R, Fassan M, Volinia S, O'Hara B, Liu CG, Palazzo JP, Gardiman M, Rugge M, Gomella LG, Croce CM and Rosenberg A: MicroRNA expression profiling of human metastatic cancers identifies cancer gene targets. J Pathol 219: 214-221, 2009.

30. Qian B, Wang DM, Gu XS, Zhou K, Wu J, Zhang CY and He XY: LncRNA H19 serves as a ceRNA and participates in non-small cell lung cancer development by regulating microRNA-107. Eur Rev Med Pharmacol Sci 22: 5946-5953, 2018. 
31. Philpott C, Tovell H, Frayling IM, Cooper DN and Upadhyaya M: The NF1 somatic mutational landscape in sporadic human cancers. Hum Genomics 11: 13, 2017.

32. Rahman MA, Barger JF, Lovat F, Gao M, Otterson GA and Nana-Sinkam P: Lung cancer exosomes as drivers of epithelial mesenchymal transition. Oncotarget 7: 54852-54866, 2016.

33. You J, Li M, Cao LM, Gu QH, Deng PB, Tan Y and Hu CP: Snaill-dependent cancer-associated fibroblasts induce epithelial-mesenchymal transition in lung cancer cells via exosomes. QJM 112: 581-590, 2019.

34. He S, Li Z, Yu Y, Zeng Q, Cheng Y, Ji W, Xia W and Lu S: Exosomal miR-499a-5p promotes cell proliferation, migration and EMT via mTOR signaling pathway in lung adenocarcinoma. Exp Cell Res 379: 203-213, 2019.

35. Verset L, Tommelein J, Moles Lopez X, Decaestecker C, Boterberg T, De Vlieghere E, Salmon I, Mareel M, Bracke M, De Wever $O$ and Demetter P: Impact of neoadjuvant therapy on cancer-associated fibroblasts in rectal cancer. Radiother Oncol 116: 449-454, 2015.
36. Wei M, Yang T, Chen X, Wu Y, Deng X, He W, Yang J and Wang Z: Malignant ascites-derived exosomes promote proliferation and induce carcinoma-associated fibroblasts transition in peritoneal mesothelial cells. Oncotarget 8: 42262-42271, 2017.

37. Zheng X, Bahr M and Doeppner TR: From tumor metastasi towards cerebral ischemia-extracellular vesicles as a general concept of intercellular communication processes. Int J Mol Sci 20: pii: E5995, 2019.

38. Ning $\mathrm{X}$, Zhang H, Wang $\mathrm{C}$ and Song $\mathrm{X}$ : Exosomes released by gastric cancer cells induce transition of pericytes into cancer-associated fibroblasts. Med Sci Monit 24: 2350-2359, 2018

This work is licensed under a Creative Commons Attribution-NonCommercial-NoDerivatives 4.0 International (CC BY-NC-ND 4.0) License. 